\title{
Rhizobium etli CE3 Carries vir Gene Homologs on a Self-Transmissible Plasmid
}

\author{
M. A. Bittinger, ${ }^{1,2}$ J. A. Gross, ${ }^{1}$ J. Widom,${ }^{3}$ J. Clardy, ${ }^{3}$ and J. Handelsman ${ }^{1}$ \\ ${ }^{1}$ Department of Plant Pathology, University of Wisconsin-Madison, Madison 53706, U.S.A.; \\ ${ }^{2}$ Program in Cellular and Molecular Biology, University of Wisconsin-Madison, Madison 53706, U.S.A.; \\ ${ }^{3}$ Department of Chemistry and Chemical Biology, Cornell University, Ithaca, NY 14853, U.S.A. \\ Accepted 15 May 2000.
}

\begin{abstract}
RosR is a transcriptional regulator important for determining cell-surface characteristics and nodulation competitiveness in Rhizobium etli CE3. We identified a 15-kb region that contains genes with similarity to members of the virB, virC, virG, and virE operons of Agrobacterium tumefaciens and demonstrated that RosR directly regulates one operon in this region. These genes were located on plasmid pa of $R$. etli CE3, which is self-transmissible between $R$. etli and $A$. tumefaciens.
\end{abstract}

ros $R$ is an important determinant of the cell surface and nodulation competitiveness in Rhizobium etli (Bittinger et al. 1997). $\operatorname{ros} R$ is a member of a family of transcriptional regulators that includes ros from Agrobacterium tumefaciens and MucR from Sinorhizobium meliloti (Cooley et al. 1991; Keller et al. 1995). ros is encoded by a chromosomal locus and specifically represses expression of the virulence operons $\operatorname{vir} C$ and virD on the Ti plasmid. mucR in S. meliloti affects the cell surface of $S$. meliloti by repressing expression of genes involved in production of galactoglucan, an alternative exopolysaccharide.

We previously identified a wide array of genes that are transcriptionally regulated by RosR, and one of the RosRregulated regions that we identified in $R$. etli CE3 displayed sequence similarity to the virCl and virD3 genes of A. tumefaciens. $R$. etli mutant MB043 contains a transposon insertion in a region with sequence similarity to the virCl and virD3 genes of A. tumefaciens (Bittinger and Handelsman 2000). The presence of vir gene homologs in a rhizobial species suggests intriguing possibilities about the evolution and ecology of the symbionts and therefore warranted further study.

In this study, we sequenced $15 \mathrm{~kb}$ of the region flanking this transposon insertion, and found that it contained homologs of other vir genes of A. tumefaciens (Fig. 1). At one end of this region, we identified open reading frames (ORFs) that encode deduced proteins that are $76 \%$ identical over 247 amino acids to VirB8, 87\% identical over 275 amino acids to VirB9, 67\% identical over 406 amino acids to VirB10, and 79\% identical

Corresponding author: Jo Handelsman, Department of Plant Pathology, University of Wisconsin-Madison, 1630 Linden Drive, Madison 53706, U.S.A; Telephone: 1-608-263-8783; Fax: 1-608-262-8643; E-mail: joh@plantpath.wisc.edu over 343 amino acids to VirB11. Downstream of the virB region is an ORF encoding a deduced protein $85 \%$ identical over 249 amino acids to VirG (86\% identical over 241 amino acids if translation initiates at the TTG codon 228 bp downstream of the ATG codon, which has been observed in $A$. tumefaciens; Pazour and Das 1990). Convergently oriented to the virG homolog are two ORFs that encode deduced proteins that are $83 \%$ identical over 230 amino acids to VirC1 and $65 \%$ identical over 184 amino acids to VirC2, respectively. The region with nucleotide similarity to the virD3 gene is not contained in a single ORF, suggesting that it is not a functional gene. ORFs encoding deduced proteins $76 \%$ identical over 63 amino acids to VirE1, 70\% identical over 557 amino acids to VirE2, and 51\% identical over 684 amino acids to VirE3 are located at the other end of the region sequenced. Between the $\operatorname{vir} C$ and virE operons is a region with sequence similarity to various insertion elements, including ISRI2, ISRm3, ISRm101, and RmInt1.

We determined that rosR, like its homolog ros in A. tumefaciens, is located on the bacterial chromosome, while the vir genes it regulates are plasmid-borne. The physical locations of the ros $R$ gene and the vir genes in the $R$. etli CE3 genome were identified by Southern blot analysis. We separated the chromosome and plasmids of $R$. etli CE3 by gel electrophoresis with a modified Eckhardt procedure (Eckhardt 1978; Hynes and McGregor 1990), transferred the DNA to membranes, and probed with DNA corresponding to the virG ORF (polymerase chain reaction [PCR] amplified with the primers 5'TTT GTC GAC GAT GCT GCT TTG CGG CCC AAC and 5'TTT GAG CTC GTT TAG CAA GGC TTC CCA AAG) or rosR ORF (amplified with the primers 5'GAT AGC GCC CGA AAA CGC T and 5'GAA AAC ATA TGA CGG ATA TGG CG) (Fig. 2). In addition to $R$. etli CE3, we analyzed the A. tumefaciens strain GMI9023 and a derivative of GMI9023 that carries the plasmid pa from $R$. etli CFN42, the parent strain of CE3 (Flores et al. 1987). The labeled DNA probe specific for the virG ORF hybridized to the band corresponding to plasmid pa, the smallest of the $R$. etli plasmids, while the probe specific for $\operatorname{ros} R$ hybridized to the chromosomal DNA that remained in the well of the gel.

We determined that plasmid pa was self-transmissible and readily transmitted between $R$. etli and $A$. tumefaciens. When R. etli MB043, which harbors a spectinomycin resistance gene 


\section{A. tumefaciens}
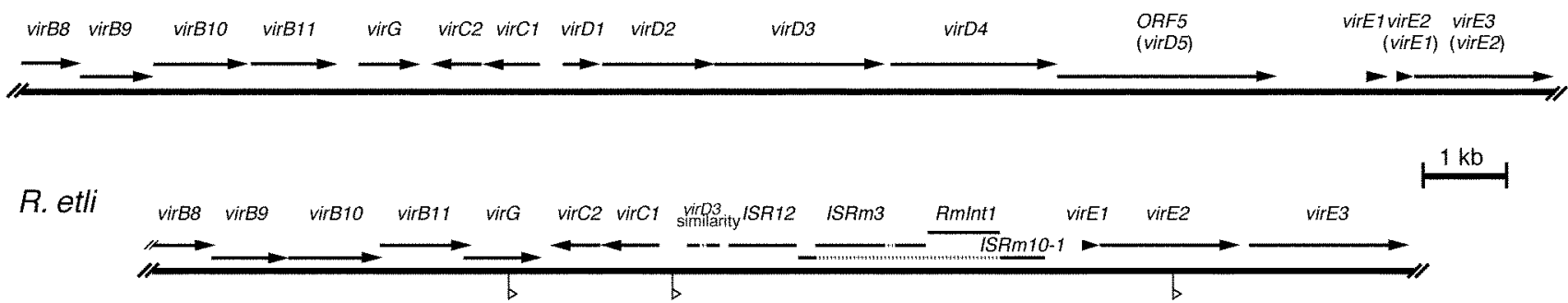

Fig. 1. The vir region of Agrobacterium tumefaciens and Rhizobium etli. Top of figure: diagram of approximately $18 \mathrm{~kb}$ of vir region of A. tumefaciens C58 (sequence from GenBank no. J03320) (Rogowsky et al. 1990). Gene names in parentheses indicate nomenclature in other Agrobacterium spp. Beneath A. tumefaciens diagram is corresponding 15-kb vir region of $R$. etli (GenBank no. AF176227). Regions with similarity to sequences in data bases are indicated with appropriate name above the region. Open reading frames are indicated by arrows; sites of promoterless gusA insertions are indicated, with orientation of gusA indicated by direction of the flag.

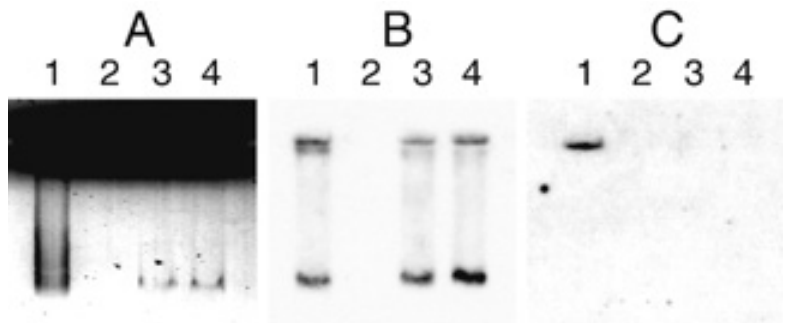

Fig. 2. Southern blot analysis of bacterial chromosome and plasmids of Rhizobium etli and Agrobacterium tumefaciens. Strains analyzed: (1) CE3; (2) GMI9023; (3) GMI9023pCFN42a; (4) GMI9023p43a. A, Gel stained with Sybr green after electrophoresis. Upper portion of panel is black due to intense staining of chromosomal DNA in the wells of the gel. This gel was blotted and probed with $(\mathbf{B}) \operatorname{vir} G$, and then $(\mathbf{C})$ stripped and reprobed with rosR. Hybridization of probes was visualized with the DIG Luminescent Detection Kit (Roche, Indianapolis, IN).

on plasmid pa, was mixed with A. tumefaciens GMI9023, spectinomycin-resistant derivatives of GMI9023 arose at a frequency of $4.4( \pm 0.4) \times 10^{-4}$, which is similar to previously reported frequencies of transfer (Noel et al. 1984). The presence of plasmid pa in the transconjugants was confirmed by Southern analysis (Fig. 2). Additionally, GMI9023 carrying only plasmid pa transmitted spectinomycin resistance back to $R$. etli (data not shown), indicating that plasmid pa is selftransmissible between members of this bacterial family.

Purified RosR protein specifically bound DNA sequences in the region upstream of the virCl ORF, further demonstrating the functional similarities between RosR and Ros. The 162-bp region immediately upstream of the virC ORF was generated by PCR (corresponding to nucleotides 6035 to 6196 of GenBank no. AF176227), labeled with digoxigenin, and used as a substrate for in vitro gel mobility shift assays with the DIG Luminescent Detection Kit (Roche, Indianapolis, IN) to visualize the labeled DNA. RosR protein was purified from Escherichia coli with the IMPACT-T7 kit (New England Biolabs, Beverly, MA) (Bittinger 1999). The mobility of this 162bp fragment decreased in the presence of purified RosR and an excess of unlabeled competitor DNA, and the amount of the shifted DNA fragment was dependent on the amount of RosR added to the binding reaction (Fig. 3 ).

To determine whether these vir genes are involved in the symbiosis of $R$. etli CE3 with common bean, we constructed mutants of CE3 with either the virG or virE2 genes disrupted, and screened those mutants for altered symbiotic phenotypes.

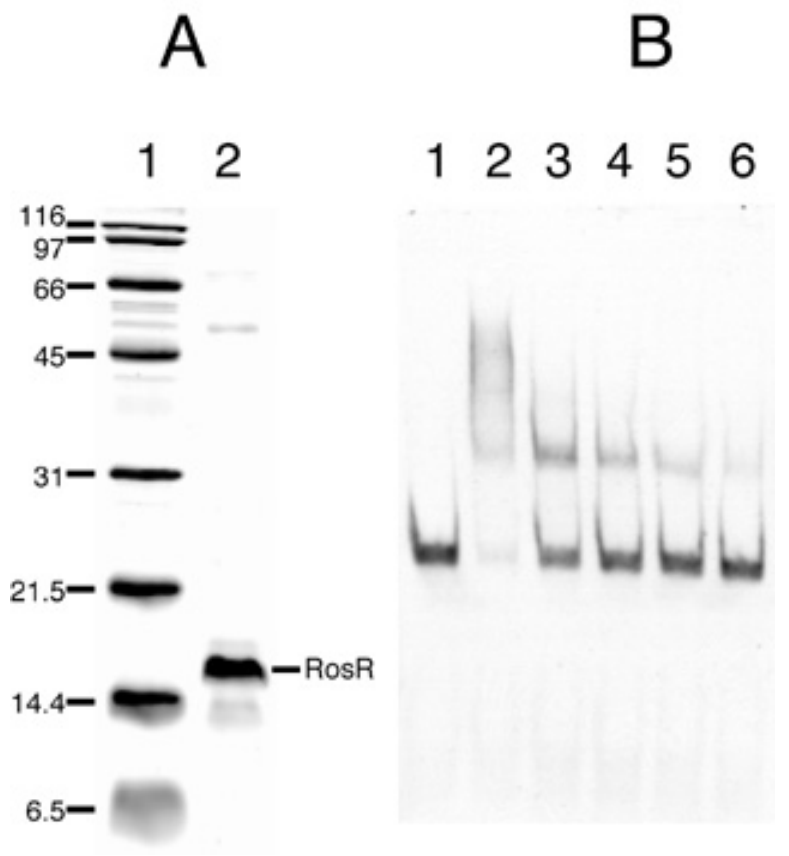

Fig. 3. Gel mobility shift assay. A, Sodium dodecyl sulfate-polyacrylamide gel electrophoresis gel of purified RosR protein stained with SYPRO Orange. Lane 1, protein standards (sizes indicated in $\mathrm{kDa}$ ); lane 2, $500 \mathrm{ng}$ of purified RosR protein. B, Electrophoretic gel mobility shift assay with purified RosR protein and labeled 162-bp DNA fragment from upstream of virC1. Lane 1, no RosR protein; lane 2, $200 \mathrm{ng}$ of RosR protein; lane 3, $100 \mathrm{ng}$ of RosR protein; lane 4, $50 \mathrm{ng}$ of RosR protein; lane 5, $25 \mathrm{ng}$ of RosR protein; lane 6, $12.5 \mathrm{ng}$ of RosR protein.

A promoterless gusA gene was introduced into the ORF of each of these genes by marker exchange with the counterselectable suicide vector pJQ200KS (Quandt and Hynes 1993) (Fig. 1). Southern analysis of genomic DNA from these mutants confirmed the gene disruptions (Bittinger 1999). Both mutants formed nitrogen-fixing nodules on Phaseolus vulgaris cv. Black Turtle, and neither mutant displayed altered nodulation competitiveness when coinoculated in a 1:1 ratio with $R$. etli CE3013, a kanamycin-resistant derivative of CE3 unaffected in competitiveness (Beattie and Handelsman 1993; Bittinger 1999).

The findings of this study reinforce the similarity between the role of RosR in $R$. etli CE3 and that of Ros in A. tumefaci- 
ens. Additionally, this is the first report of a Rhizobium species carrying homologs of the vir genes. Although it is surprising to find homologs of the vir genes in a rhizobial species, they may no longer be involved in pathogenesis due to the transposition of the large insertion element. Because plasmid pa is self-transmissible, it seems likely that it is present as a result of horizontal transfer from other members of the Rhizobiaceae; however, we do not know if this plasmid provides a selective advantage to the bacterium in its saprophytic life in the soil. It would be interesting to identify the other genes present on this plasmid and ultimately determine whether the rhizobial vir homologs can functionally complement vir mutants of A. tumefaciens.

\section{LITERATURE CITED}

Beattie, G. A., and Handelsman, J. 1993. Evaluation of a strategy for identifying nodulation competitiveness genes in Rhizobium leguminosarum biovar phaseoli. J. Gen. Microbiol. 139:529-538.

Bittinger, M. A. 1999. The RosR regulon of Rhizobium etli. Ph.D. diss. University of Wisconsin-Madison, Madison.

Bittinger, M. A., and Handelsman, J. 2000. Identification of genes in the RosR regulon of Rhizobium etli. J. Bacteriol. 182:1706-1713.

Bittinger, M. A., Milner, J. L., Saville, B. J., and Handelsman, J. 1997. rosR, a determinant of nodulation competitiveness in Rhizobium etli. Mol. Plant-Microbe Interact. 10:180-186.

Cooley, M. B., D'Souza, M. R., and Kado, C. I. 1991. The virC and virD operons of the Agrobacterium Ti plasmid are regulated by the ros chromosomal gene: Analysis of the cloned ros gene. J. Bacteriol. 173:2608-2616.

Eckhardt, T. 1978. A rapid method for the identification of plasmid deoxyribonucleic acid in bacteria. Plasmid 1:584-588.

Flores, M., Gonzalez, V., Brom, S., Martinez, E., Pinero, D., Romero, D., Davila, G., and Palacios, R. 1987. Reiterated DNA sequences in Rhizobium and Agrobacterium spp. J. Bacteriol. 169:5782-5788.

Hynes, M. F., and McGregor, N. F. 1990. Two plasmids other than the nodulation plasmid are necessary for formation of nitrogen-fixing nodules by Rhizobium leguminosarum. Mol. Microbiol. 4:567-74.

Keller, M., Roxlau, A., Weng, W. M., Schmidt, M., Quandt, J., Niehaus, K., Jording, D., Arnold, W., and Pühler, A. 1995. Molecular analysis of the Rhizobium meliloti mисR gene regulating the biosynthesis of the exopolysaccharides succinoglycan and galactoglucan. Mol. PlantMicrobe Interact. 8:267-277.

Noel, K. D., Sanchez, A., Fernandez, L., Leemans, J., and Cevallos, M. A. 1984. Rhizobium phaseoli symbiotic mutants with transposon $T n 5$ insertions. J. Bacteriol. 158:148-155.

Pazour, G. J., and Das, A. 1990. VirG, an Agrobacterium tumefaciens transcriptional activator, initiates translation at a UUG codon and is a sequence-specific DNA-binding protein. J. Bacteriol. 172:1241-1249.

Quandt, J., and Hynes, M. F. 1993. Versatile suicide vectors which allow direct selection for gene replacement in Gram-negative bacteria. Gene 127:15-21.

Rogowsky, P. M., Powell, B. S., Shirasu, K., Lin, T. S., Morel, P., Zyprian, E. M., Steck, T. R., and Kado, C. I. 1990. Molecular characterization of the vir regulon of Agrobacterium tumefaciens: Complete nucleotide sequence and gene organization of the $28.63-\mathrm{kbp}$ regulon cloned as a single unit. Plasmid 23:85-106. 